\title{
Laparoscopic Treatment of Colonic Intussusception Caused by Sigmoid Colon Cancer
}

\author{
Hee Yong Kwak, M.D., Ph.D., Min Sung Kim, M.D., Jin Wan Park, M.D., Dong Hee Kim, M.D., Ph.D., Joo-Ho Lee, M.D., Ph.D., \\ Yeon Soo Chang, M.D., Ph.D. \\ Department of Surgery, Nowon Eulji University Hospital, Eulji University College of Medicine, Seoul, Korea
}

\begin{abstract}
Reports on the laparoscopic treatment for colonic intussusception are exceedingly rare. We report a case of colonic intussusception caused by sigmoid colon cancer which was treated with a laparoscopic approach. A 76-year-old man visited an emergency room with the chief complaint of lower abdominal pain. He was diagnosed with colonic intussusception probably due to sigmoid colon cancer on a CT scan. Upon laparoscopic exploration, sigmoid colon intussusception was noted. Manual reduction was impossible because the colonic walls were friable and due to the possibility of a cancerous leading point. Therefore, the bowel was resected with en bloc Hartmann procedure. Pathology of the resected specimen revealed a tumor measuring $4.5 \mathrm{~cm}$ in size and comprising moderately differentiated adenocarcinoma (pT3N0M0, pStage II). The patient's postoperative course was uneventful and was discharged on the 8th day after surgery.
\end{abstract}

Keywords: Intussusception, Laparoscopy, Colon cancer

Supplementary video file: This article contains supplementary material (https://doi.org/10.7602/ jmis.2020.23.3.149).

This is an Open Access article distributed under the terms of the Creative Commons Attribution Non-Commercial License (http:// creativecommons.org/licenses/by-nc/4.0/) which permits unrestricted non-commercial use, distribution, and reproduction in any medium, provided the original work is properly cited.

\author{
Received April 24, 2020 \\ Revised June 4, 2020 \\ Accepted June 8, 2020 \\ Corresponding author \\ Yeon Soo Chang \\ Department of Surgery, Nowon Eulji \\ University Hospital, Eulji University \\ College of Medicine, 68 Hangeulbiseok- \\ ro, Nowon-gu, Seoul 01830, Korea \\ Tel: $+82-2-970-8688$ \\ Fax: +82-2-970-8227 \\ E-mail: cutdowngs@naver.com \\ ORCID: \\ https://orcid.org/0000-0002-6570-5920
}

Copyright $\odot 2020$ The Journal of Minimally Invasive Surgery. All rights reserved.

\section{INTRODUCTION}

Intussusception is the prolapse of a bowel loop with its mesenteric fold into the lumen of a contiguous segment causing intestinal obstruction. The majority of intussusceptions are ileocolic, while the remaining are ileoileal or colocolic type. ${ }^{2}$ Any intestinal condition that changes the normal pattern of peristalsis increases the risk of intussusception. ${ }^{3}$ The optimal management strategy for adult intussusception remains controversial; however, a surgical approach is usually chosen as there is always a leading point unlike in children. Although most approaches are undertaken via an open conventional method, we report a case of colonic intussusception caused by colon cancer which was treated with a laparoscopic approach.

\section{PROCEDURE}

A 76-year-old man visited the emergency room due to periumbilical and suprapubic pain experienced for a day after the consumption of breakfast. The score on the Numeric rating pain scale (NRS) was recorded as 4 . The patient displayed no other gastrointestinal symptoms, such as nausea, vomiting, diarrhea, melena, weight loss, or abdominal distension. The patient had been under regular medication for hypertension. He had a history of laparoscopic cholecystectomy 9 years ago for cholecystitis. Upon initial physical examination, mild tenderness was noted on 


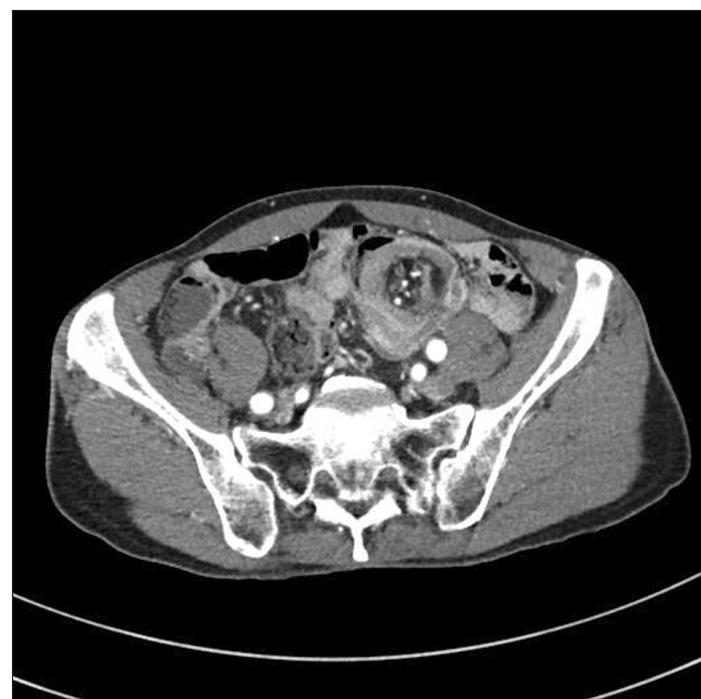

Fig. 1. CT scan showed 'a target sign' appearance confirming the diagnosis of intussusception probably due to proximal sigmoid colon cancer with mild pelvic ascites.

the left lower quadrant and suprapubic area; however, the symptom was observed to subside after pain control. Hematological laboratory examination findings were within the normal range. CT scan revealed 'a target sign' appearance confirming the diagnosis of intussusception probably due to proximal sigmoid colon cancer with mild pelvic ascites (Fig. 1). There was no evidence of enlarged lymph nodes.

Laparoscopic en bloc Hartmann procedure for colon cancer was performed (Fig. 2). A camera port was inserted at the site of the umbilicus $(12 \mathrm{~mm})$ with three working ports at right lower quadrant $(12 \mathrm{~mm})$, right medial quadrant $(12 \mathrm{~mm})$, and left lower quadrant $(5 \mathrm{~mm})$, respectively. The lesion was $5 \times 5 \mathrm{~cm}$ sized and was located at AV $40 \mathrm{~cm}$ above the peritoneal reflection and no serosa infiltration or adjacent organ invasion was noted grossly. Vessel ligation was performed at the level of the root of the superior rectal artery and the left colic artery was preserved. The hypogastric nerve was completely preserved and lymph nodes dissection around root of inferior mesenteric artery was done. Both the proximal and distal colonic segments were resected with Echelon 60 stapling device (Ethicon Endo-surgery; Johnson \& Johnson, Cincinnati, OH, USA) with gold cartridges (compressible thickness to $3.6 \mathrm{~mm})(\times 2)$ and the specimen was retracted through the site for colostomy. The patient recovered without any postoperative complications.

The pathologic finding was fungating and polypoid moderately differentiated adenocarcinoma at the sigmoid colon sized $4.5 \times 2.5 \times 1.5 \mathrm{~cm}$ which invaded the subserosa or pericolic adipose tissue (pT3). The resection margins were free from carcinoma and no metastasis in 17 regional lymph nodes was noted (pN0).

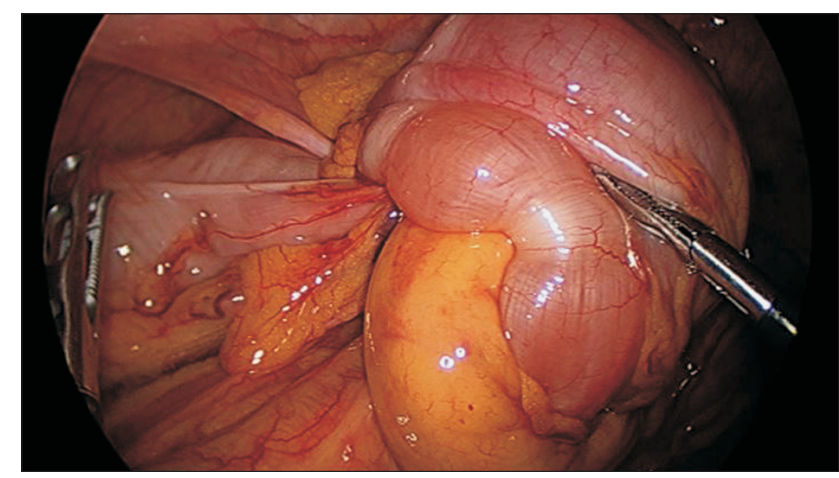

Fig. 2. Intraoperative findings: The intussusception of sigmoid colon was observed at AV $40 \mathrm{~cm}$ above the peritoneal reflection. The lesion was $5 \times 5 \mathrm{~cm}$ sized and no serosa infiltration or adjacent organ invasion was noted grossly.

The patient was discharged on the 8th day after surgery.

He received 12 cycles of chemotherapy with FOLFOX regimen, and Hartmann reversal operation was done after the end of chemotherapy.

This case report was approved by the Institutional Review Board of Nowon Eulji University Hospital (EMCS 2020-06-001).

\section{DISCUSSION}

Intussusception is the prolapse of a bowel loop with its mesenteric fold into the lumen of a subsequent segment in a telescopic fashion causing intestinal obstruction. ${ }^{1}$ Unlike in children, intussusception is a rare cause of obstruction in adults. The majority of adult intussusception is treated with surgical management by a conventional open method. In this study, we report a rare case of a colocolic type intussusception treated with laparoscopic approach.

Pediatric intussusception is treated initially with nonoperative air reduction and surgical manual reduction is performed in case of failure in air reduction. This is because the most common cause of intussusception is idiopathic in childhood, ${ }^{4}$ while most adult patients have an underlying pathologic leading point. ${ }^{5,6}$ Therefore, the management of intussusception differs between pediatric and adult patients. Adult intussusception is usually treated with surgical exploration and resection of the bowel.

Adult intussusception can be classified according to its location. Most of the intussusceptions are ileocolic and the rest are the ileoileal or the colocolic types. ${ }^{2}$ Malignant tumors located in the enteric site are the most common leading points in histologic reviews. Primary adenocarcinoma, followed by lymphoma and metastatic carcinoma, are the main causes of colonic intussusception. ${ }^{7}$ Hence, in the case of colonic intussusception, en bloc resection without reduction should be performed in order to avoid 
potential intraluminal seeding or venous tumor dissemination.

The CT scan is the most accurate preoperative diagnostic method. ${ }^{8}$ In this present case, CT showed a typical bowel-withinbowel appearance, which is called 'a target sign' appearance, confirming the diagnosis of intussusception. The CT scan is superior to other imaging techniques, such as ultrasonography or small bowel series, in that it helps not only in the diagnosis of intussusception but also with the differentiation from other abdominal emergencies.

When a bowel obstruction occurs, bowel edema develops and little space is left in the abdominal cavity. Therefore, emergent surgical exploration by an open conventional method is usually chosen in most cases. In the present case, the patient with colocolic intussusception was treated with a laparoscopic approach, which is rarely reported for colonic intussusception. ${ }^{9}$ Dell'abate et al. ${ }^{10}$ also have reported a case of laparoscopic management of sigmoid colon intussusception by a malignant tumor. However, to our knowledge; there are few reports of laparoscopic treatment of intussusception caused by sigmoid colon cancer in Asian patients, who have a smaller capacity of abdominal volume than patients of western countries. In our case, preoperative CT scan and intraoperative findings demonstrated little bowel edema and dilatation and no severe adhesion, we decided laparoscopic approach. Notably, additional cases should be reviewed to confirm the feasibility of the laparoscopic approach in colonic intussusception.

In conclusion, intussusception is an uncommon cause of bowel obstruction in adults which could be cumbersome to identify. Accurate diagnoses with CT scans and prompt surgical management are necessary for intussusception. Although an open conventional method is commonly performed, the laparoscopic approach could be feasible in selected cases.

\section{ORCID}

Hee Yong Kwak, https://orcid.org/0000-0002-4252-6751

Min Sung Kim, https://orcid.org/0000-0001-6370-7239

Jin Wan Park, https://orcid.org/0000-0002-3816-8357

Dong Hee Kim, https://orcid.org/0000-0001-9910-8414

Joo-Ho Lee, https://orcid.org/0000-0001-8499-664X

Yeon Soo Chang, https://orcid.org/0000-0002-6570-5920

\section{AUTHORS' CONTRIBUTIONS}

Conceptualization: Dong Hee Kim. Formal analysis: Jin Wan
Park and Joo-Ho Lee Methodology: Min Sung Kim and Hee Yong Kwak. Writing-original draft: Hee Yong Kwak, Yeon Soo Chang Writing-review and editing: Yeon Soo Chang and Min Sung Kim.

\section{CONFLICT OF INTEREST}

None.

\section{FUNDING}

None.

\section{ACKNOWLEDGMENTS}

None.

\section{REFERENCES}

1) Wilson A, Elias G, Dupiton R. Adult colocolic intussusception and literature review. Case Rep Gastroenterol 2013;7:381-387.

2) Catalano O. Transient small bowel intussusception: CT findings in adults. Br J Radiol 1997;70:805-808.

3) Weilbaecher D, Bolin JA, Hearn D, Ogden W, 2nd. Intussusception in adults. Review of 160 cases. Am J Surg 1971;121:531-535.

4) Arbizu RA, Aljomah G, Kozielski R, Baker SS, Baker RD. Intussusception associated with adenovirus. J Pediatr Gastroenterol Nutr 2014;59:e41.

5) Begos DG, Sandor A, Modlin IM. The diagnosis and management of adult intussusception. Am J Surg 1997;173:88-94.

6) Azar T, Berger DL. Adult intussusception. Ann Surg 1997;226:134138.

7) Hong KD, Kim J, Ji W, Wexner SD. Adult intussusception: a systematic review and meta-analysis. Tech Coloproctol 2019;23:315-324.

8) Zubaidi A, Al-Saif F, Silverman R. Adult intussusception: a retrospective review. Dis Colon Rectum 2006;49:1546-1551.

9) Kyo K, Azuma M, Okamoto K, et al. Laparoscopic resection of adult colon duplication causing intussusception. World J Gastroenterol 2016;22:2398-2402.

10) Dell'abate P, Del Rio P, Sommaruga L, Arcuri MF, Sianesi M. Laparoscopic treatment of sigmoid colon intussusception by large malignant tumor. Case report. G Chir 2009;30:374-376. 